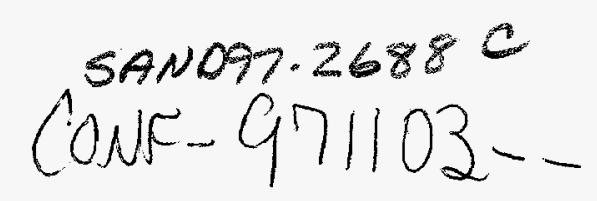

\title{
Wire Number Doubling in Plasma-Shell Regime Increases Z-Accelerator X-Ray Power*
}

\author{
T. W. L. Sanford**, R. B. Spielman, G. A. Chandler, D. L. Fehl, \\ W. A. Stygar, K. W. Struve, C. Deeney, T. J. Nash, J. F. Seamens, \\ R. C. Mock, T. L. Gilliland, J. S. McGurn, D. O. Jobe,
}

S. P. Breeze, and M. F. Vargas

RECEIVED

NOV $2+1997$

Sandia National Laboratories, P.O. Box 5800, Albuquerque, NM 87185 USA

OST I

\begin{abstract}
Doubling the number of tungsten wires from 120 to 240 , keeping the mass fixed, increased the radiated $x$-ray power relative to the electrical power at the insulator stack of the $z$ accelerator by $(40 \pm 20) \%$ for 8.75 - and 20 -mm-radii z-pinch wire arrays. Radiation-magneto-hydrodynamic calculations suggest that the arrays were operating in the "plasma shell" regime, where the plasma generated by the individual wires merge prior to the inward implosion of the entire array.
\end{abstract}

Sandia is a multiprogram laboratory operated by Sandia Corporation, a Lockheed Martin Company, for the United States Department of Energy under contract DE-ACO4-94AL85000.

\section{DSSTRIBUTION OF THIS DOCUMENT IS UNLIMIED}

* Work supported by U.S. DOE Contract No. DE-AC04-94AL85000

** Paper presented at 39th Annual Meeting APS Division of Plasma Physics, Nov. 17-21, 1997. Pittsburgh, PA. 


\section{DISCLAIMER}

This report was prepared as an account of work sponsored by an agency of the United States Government. Neither the United States Government nor any agency thereof, nor any of their employees, make any warranty, express or implied, or assumes any legal liability or responsibility for the accuracy, completeness, or usefulness of any information, apparatus, product, or process disclosed, or represents that its use would not infringe privately owned rights. Reference herein to any specific commercial product, process, or service by trade name, trademark, manufacturer, or otherwise does not necessarily constitute or imply its endorsement, recommendation, or favoring by the United States Government or any agency thereof. The views and opinions of authors expressed herein do not necessarily state or reflect those of the United States Government or any agency thereof. 


\section{DISCLAmIER}

Portions of this docoment may be illegible in electronic image products. Images are produced from the best available original document 


\section{INTRODUCTION}

Increasing wire number by an order-of-magnitude from tens of wires to greater than 100 (by simultaneously decreasing the interwire gap and wire size in order to maintain a constant array mass) has resulted in the highest total $\mathrm{x}$-ray power and narrowest pulse width measured for aluminum-wire array z-pinch implosions [1] on the 20-TW Saturn accelerator [2]. Radiationmagneto-hydrodynamic-code (RMHC) calculations suggest that for the very high number of wires used, the individual wire plasmas merge to form a plasma shell prior to implosion of the array [1, 3]. In this "plasma-shell" regime, arrays with 90 to 192 aluminum wires (interwire gaps 0.6 to 0.4 $\mathrm{mm}$ and wire diameters 12.7 to $10 \mu \mathrm{m}$ ) produced peak total radiated powers of 30 to 40 TW with pulse widths (FWHM) as short as 4 to 5 ns. Subsequently, on Saturn, application of this load technique utilizing very large-numbers of tungsten wires and utilizing a simultaneous increase in implosion velocity (by reducing load mass and increasing load radius) has lead to a similar reduction in pulse width [4]. Specifically, using arrays of 120 wires and increasing the implosion velocity to $65 \mathrm{~cm} / \mu \mathrm{s}$, yielded radiated powers of $65-85 \mathrm{TW}$ and pulse widths of $4 \mathrm{~ns}$. As expected, the total power radiated was higher than that achieved with aluminum, since there are substantially more radiative transitions available in tungsten relative to aluminum.

Because of the successful application of very high-wire-number loads to the generation of high radiated power on Saturn and because the technology of constructing these loads has since improved, the loads designed for initial use on the $\mathrm{Z}$ accelerator $[5,6]$ employed arrays with at least 120 wires. Moreover, experiments on Saturn showed that with such high-wire-number arrays, high-quality implosions could also be achieved with arrays having initial radii as large as $20 \mathrm{~mm}$ [7]. Use of such loads thus permitted the quality of the implosion to be maintained, while the large radius minimized the initial electrical stress on the $\mathrm{Z}$ accelerator due to load inductance, as the power flow in the upstream portion of the accelerator was improved.

In this paper, the increase in peak total radiated power with wire number is discussed for the initial shots on the $\mathrm{Z}$ accelerator. Because the power flow within the accelerator was modified 
during this shot sequence, the radiated powers are normalized to the power measured at the insulator stack $[8,9]$.

\section{EXPERIMENTAL ARRANGEMENT}

The peak stack power was measured using B-dot and D-dot detectors [8]. This power varied from 37 to $45 \mathrm{TW}[8,9]$. Owing to the calibration uncertainties of the detectors, the estimated uncertainty of the stack power is $\sim 10 \%$. The peak total radiated $x$-ray power is inferred from a spectroscopic unfold $[10,11]$ of data taken using five filtered $\mathrm{x}$-ray diode (XRD) detectors [12] and

a Lambertian-emission model, which permitted the $\mathrm{x}$-ray energies to be distinguished in five overlapping channels between 150 and $2300 \mathrm{eV}$ [6]. The uncertainty in the peak radiated power, due to XRD calibration and unfold uncertainties, is estimated to be $\pm 20 \%$. The shot-to shot variation in the radiated power for similar conditions is typically $\pm 13 \%$. The associated variation in the width of the radiation pulse is measured in the lowest energy channel (the XRD filtered by 5 $\mu \mathrm{m}$ of Kimfol, which best represents the shape of the total radiated power pulse) and is typically $\pm 7 \%$. These values are those used for the uncertainties shown in the figures.

Details of the accelerator, load, and diagnostic layout are given in Refs. 5, 6, 8, 9, and 13-14. For the data discussed here, two load geometries each having $20-\mathrm{mm}$ long tungsten wires were used (Fig. 1A). In one, the radius of the wires was fixed at $20 \mathrm{~mm}$ and in the other the radius was reduced to $8.75 \mathrm{~mm}$. For both arrays, the anode-cathode spacing between the wires and the current-return shell was $5 \mathrm{~mm}$. Figure $1 \mathrm{~B}$ shows a photograph of one of the 240 -wire, $20-\mathrm{mm}$ radius loads and illustrates the characteristic uniformity of the wires fielded.

\section{MEASUREMENTS}

Figure $2 \mathrm{~A}$ plots the results of the initial mass scan using the large-radius array, where the number of wires was fixed at 120 wires. A mass of $4 \mathrm{mg}$ optimizes the peak radiated power and is consistent with that expected from a circuit-code analysis of optimal energy coupling to the load [6]. The width of the radiated power pulse (Fig. 2B) approximately tracks the inverse of the peak radiated power as might be expected by assuming that the bulk of the energy is radiated prior to the 
expansion of the plasma after stagnation. Shown also in Figs. 2A and $2 \mathrm{~B}$ are the peak power and pulse width measured when the number of wires is doubled, respectively. Comparison of the 120 wire shots and the 240 -wire shots indicates that doubling the wire number increases the radiated power by $(32 \pm 22) \%$ and reduces the pulse width by $(16 \pm 10) \%$. For these two types of shots, the interwire spacing decreased from 1.05 to $0.52 \mathrm{~mm}$, and the individual wire diameter from $\sim 10$ (Shot \#19) to $7.5 \mu \mathrm{m}$ (Shots \#26 and \#52). The agreement in the power ratios measured between the two 240-wire Shots \#26 and \#52, which spanned the bulk of the measurements discussed here, gives confidence in the measured increase in radiated power with wire number.

Figure $3 \mathrm{~A}$ depicts the results of the mass scan using the small-radius array, where the number of wires was also changed from 120 to 160 to 240 . The relative insensitivity of the radiated power to decreasing mass is partly due to the increase in implosion velocity and the simultaneous current loss upstream of the load owing to the higher inductances generated earlier in the implosion, as is discussed in Ref. 6. Within the shot-to-shot variation, the data near a mass of $12 \mathrm{mg}$ show a monotonic increase in both power (Fig. 3A and 4A) and a decrease in pulse width (Fig. 3B and $4 \mathrm{~B})$ with an increase in wire number. Comparison of the 120 -wire shots with the 240 -wire shot indicates that doubling the wire number increases the radiated power by $(45 \pm 22) \%$ and reduces the pulse width by (29士9)\%. Over this range, the interwire spacing decreased from 0.46 to $0.23 \mathrm{~mm}$, and the wire diameter from $\sim 18$ (Shot \#27) to $13 \mu \mathrm{m}$ (Shot \#35). Together with the large-radius measurements, these data give a sublinear power-law growth in radiated power with wire number increase. This growth is less than the linear power-law dependence on wire number measured for the aluminum-wire data from Ref. 1 taken in the "plasma-shell" regime.

Also shown in Fig. 4A is the relative peak power (Shot \#33) measured when 2 wires were placed in each slot as designed for the 120-wire, small-radius arrays. Within the shot-to-shot variation, the radiated power for this special configuration shows little increase due to wire number doubling, especially when compared with Shot \#35, which employed 240 equally-spaced wires having nearly the same total mass as Shot \#33. Moreover, the pulse width associated with Shot 
$\# 33$ is $20 \%$ larger than that measured for Shot \#35 (Fig. 4B). These limited measurements suggest that reducing the interwire gap rather than wire size may be the key factor in the power increase with wire number, for these very high-wire-number arrays.

The increase in radiated power with increasing wire number for either array radius is also observed directly in the data without normalizing to the power at the insulator stack, as shown in Figs. 5A and 5B for the large and small radius arrays, respectively. The data in these figures illustrate the simultaneous reduction in pulse width with increasing wire number, giving confidence in the overall observation of a moderate gain in peak power with wire number increase, even with these already high-wire-number loads.

\section{THEORETICAL MODELING}

One-dimensional RMHC [15] calculations [16] by the late George Allshouse suggest that for these large-wire-number loads, the individual wire plasmas merge prior to the implosion of the entire array. In these calculations, the measured current for Shot \#18 (Fig. 6), which used the large radius array with a mass of $5.1 \mathrm{mg}$, was taken as input to the code. Figure 7 graphs the outside radius of the plasma shell and shows that the implosion starts at about 50 ns. For these calculations, the start time is chosen to be the time at which the 10-90\% rise time of the main current pulse extrapolates to the time axis (Fig. 6A). The precurrent pulse is shown in Fig. 6B.

The expansion and self pinching of the individual wires within the array as a function of the number of wires used in the array is also shown in Fig. 7. For these calculations, the mass per wire and the current per wire is scaled inversely with the number of wires so that the total mass remains constant at $5.1 \mathrm{mg}$ and the current sums to the total current. The marks on the left represent the half spacing between wires for the respective wire numbers. From this figure, it is apparent that $\sim 80$ wires are required for the wire plasmas to contact adjacent plasmas before selfpinching takes place. Even for the case of 500 wires, there is still a tendency for the wires to self

pinch. For more than $\sim 80$ wires, however, the self pinching takes place only after adjacent 
plasmas make contact, leading to the conclusion that the array implodes as a plasma shell instead of individual wires.

It is well known that the resistivities used in these calculations are a poor approximation to the true resistivity [17]. In order to check the sensitivity to this uncertainty, a series of calculations were run [16] where the resistivity was varied in the following ways: (1) the resistivity below $1 \mathrm{eV}$ was multiplied by 100 and above $1 \mathrm{eV}$ by a factor of two; (2) the resistivity below $1 \mathrm{eV}$ was multiplied by a factor of 0.1 and a factor of 0.5 above $1 \mathrm{eV}$; (3) the resistivity below $1 \mathrm{eV}$ was multiplied by a factor of 100 and by a factor of 0.5 above $1 \mathrm{eV}$. The results are shown in Fig. 8 for the case of 120 wires. The results are rather insensitive to the resistivity model except for model \#3. Model \#3 produces a significantly smaller expanded radius, but still more than the interwire half spacing of $0.52 \mathrm{~mm}$. For all models, the wire-plasma merger occurs $\sim 30 \mathrm{~ns}$ before the array begins to implode, suggesting that for the large-wire-number loads discussed here, the loads are likely operating in the "plasma shell regime." The model used in these calculations requires, however, that $\ln \wedge \gg>1$, where $\wedge$ is the Debye length divided by the strong-interaction distance $[15,17]$. This condition does not apply to the initial stages, where the solid wire is heated and converted to plasma. Recent calculations [18] with an improved resistivity model suggests that a large fraction of the wire remains solid until near the peak of the current pulse. The actual merger is thus a three-dimensional process, which likely significantly alters the implications of the onedimensional analysis presented here.

\section{SUMMARY}

In conclusion, although the measurements were made simultaneously with upgrades to the pulsed-power system, both the raw data and the data when normalized to the power at the insulator stack show a clear monotonic increase in the radiated power with increase in wire number and a simultaneous reduction in pulse width. For either radius load, the radiated power increase is on the order of $(40 \pm 20) \%$ when the number of wires is doubled from 120 to 240 . One-dimensional calculations suggest that the loads are operating in the "plasma-shell" regime, where the individual 
wire plasma merge prior to the over all implosion of the array. In this regime, the measured power increase with wire number is less than half that measured for the aluminum wire implosions operating in the same regime on the Saturn accelerator.

\section{ACKNOWLEDGMENTS}

We thank T. A. Haill and B. M. Marder for retrieving the Allshouse calculations and for useful discussions; J. S. DeGroot for useful discussions; the Z-operations team for technical support; M. K. Matzen, R. J. Leeper, J. E. Maenchen, and D. H. McDaniel, for vigorous programmatic support; and J. S. DeGroot, and T. A. Haill for carefully reviewing this paper. 


\section{REFERENCES}

[1] T. W. L. Sanford, et al., Phys. Rev. Lett. 77, 5063 (1996).

[2] D. D. Bloomquist, et al., Proc. 6th Int. IEEE Pulsed Power Conf. Arlington, VA. P. J. Turchi and B. H. Bernstein, ed., p. 310. New York: IEEE. 1987.

[3] B. M. Marder, T. W. L. Sanford, and G. O. Allshouse. "Numerical Simulations of Annular Wire-Array Z-Pinches in $(X, Y),(R, \Theta)$, and $(R, Z)$ Geometries." Sandia National Laboratories Technical Report, SAND97-2725, to be published (1997).

[4] C. Deeney, et al. "Power Enhancement by Increasing the Initial Radius and Wire Number of Tungsten Z Pinches." Phys. Rev. E. To be published (November 1997).

[5] R. B. Spielman, et al. Proc. of the 11th Int. Conf. On Particle Beams, K. Jungwirth and J. Ullschmied, ed. p. 150. Prague, Czech Republic. 1996.

[6] R. B. Spielman, et al. "PBFA Z: A 60-TW/5-MJ Z-Pinch Driver." Proc. 1Ith IEEE International Pulsed Power Conference to be published (1997).

[7] T. W. L. Sanford, et al. "Variation of High-Power Aluminum-Wire Array Z-Pinch Dynamics with Wire Number, Load Mass, and Array Radius.” Sandia National Laboratories Technical Report, SAND96-3031 (July 1997), AIP 4th International Conference of Dense Z-Pinches (May 28-30, 1997) to be published.

[8] W. A. Stygar, et al. "D-Dot and B-Dot Monitors for Z-Vacuum-Section Power-Flow Measurements." Proc. 11th IEEE International Pulsed Power Conference (1997) to be puslished.

[9] W. A. Stygar, et al. "Design and Performance of the $\mathrm{Z}$ Magnetically-Insulated Transmission Lines." Proc. 11 th IEEE International Pulsed Power Conference (1997) to be published.

[10] D. L. Fehl et al. Rev. Sci. Instrum. 68, 1 (1997).

[11] D. L. Fehl and F. Biggs. Rev. Sci. Instrum. 68, 890 (1997).

[12] G. A. Chandler, et al. Rev. Sci. Instrum. 63, 4828 (1992). 
[13] K. W. Struve, et al. "Design Calibration and Performance of Water Diagnostics on the PBFA Z," Mission Research Corporation Technical Report, MRC/ABQ-N-586 (September 1997). Proc. 11 th IEEE International Pulsed Power Conference (1997) to be published.

[14] K. W. Struve et al. "Circuit-Code Modeling of the PBFA Z for X-Pinch Experiments." Mission Research Corporation Technical Report, MRC/ABQ-N-587 (September 1997). Proc. 11th IEEE International Pulsed Power Conference (1997) to be published.

[15] J. H. Hammer et al. Phys. Plasmas 3, 2063 (1996).

[16] G. O. Allshouse, "Single Tungsten Wire Calculations for PBFAZ Shot \#18." Memo to T. Sanford (November 15, 1996), unpublished.

[17] J. S. DeGroot, private communication (1996).

[18] D. Reisman, private communication (1997). 


\section{FIGURE CAPTIONS}

1A. Schematic of wire-array assembly showing the 6-mm radial-feed gap narrowing to the 5-mm anodecathode gap adjacent to the wires and indicating the location of the load-current monitor (B-dot probe).

B. Photograph of one of the $20-\mathrm{mm}$ radius arrays with 240 wires.

2A. Ratio of peak total radiated power relative to peak electrical power measured at the insulator stack as a function of array mass, showing increase in radiated power near $4 \mathrm{mg}$ when the number of wires is doubled, for the $20-\mathrm{mm}$ radius array. Shot numbers are shown adjacent the shots near array masses of $4 \mathrm{mg}$.

B. Radiation pulse width as a function of array mass, showing decrease in pulse width (FWHM) near 4 $\mathrm{mg}$ when the number of wires is doubled, for the $20-\mathrm{mm}$ radius array.

3A. Ratio of peak total radiated power to peak electrical power measured at the insulator stack as a function of array mass, showing increase in radiated power when the number of wires is increased, for the $8.75-\mathrm{mm}$ radius array. Shot numbers are shown adjacent selected shots.

B. Radiation pulse width (FWHM) as a function of aray mass, showing decrease in pulse width when the number of wires is increased, for the $8.75-\mathrm{mm}$ radius array.

4A. Ratio of peak total radiated power to peak electrical power measured at the insulator stack as a function of wire number for the $8.75-\mathrm{mm}$ radius array, showing increase in radiated power with wirenumber. Shot numbers are shown adjacent selected shots.

B. Radiation pulse width (FWHM) as a function of wire number for the $8.75-\mathrm{mm}$ radius array, showing decrease in width with wire-number.

5A. Comparison of the signal from the XRD filtered by $5 \mu \mathrm{m}$ of Kimfol, for the 120-wire Shot \#19, with that measured for the 240-wire Shot \#26, for an array mass of about $4 \mathrm{mg}$, showing the increase in peak power and decreasing pulse width with wire number doubling.

B. Comparison of the signal from the XRD filtered by $5 \mu \mathrm{m}$ of Kimfol for the 120 -wire Shot \#27, with that measured for the 160-wire Shot \#34, with that measured for the 240-wire Shot \#35, at nearly 
identical array masses of $12 \mathrm{mg}$, showing the increase in peak power and decreasing pulse width with wire number increase.

6A. Main load-current pulse measured for Shot \#18, using B-dot probe shown in Fig. 1A.

B. Load-current prepulse measured for Shot \#18, using B-dot probe shown in Fig. 1A.

7. Calculated outside radius of the array as a function of time, for Shot \#18 (for array mass of $5.1 \mathrm{mg}$ and initial array radius of $20 \mathrm{~mm}$ ), showing that the implosion of the array starts at about $50 \mathrm{~ns}$. Shown also are individual wires radii calculated as a function of time that correspond to arrays with varying numbers of wire, assuming that the total mass of the array and the initial array radius remains fixed at $5.1 \mathrm{mg}$ and $20 \mathrm{~mm}$. The marks on the left side represent the half spacing for the respective wire numbers.

8. Calculated individual wire radii as a function of time and resistivity model (see text) for a 120 wirenumber, 5.1-mg-mass, and 20-mm-radius array, showing that the results are insensitive to resistivity model except for Model \#3. Half spacing between wires corresponds to $0.52 \mathrm{~mm}$. 


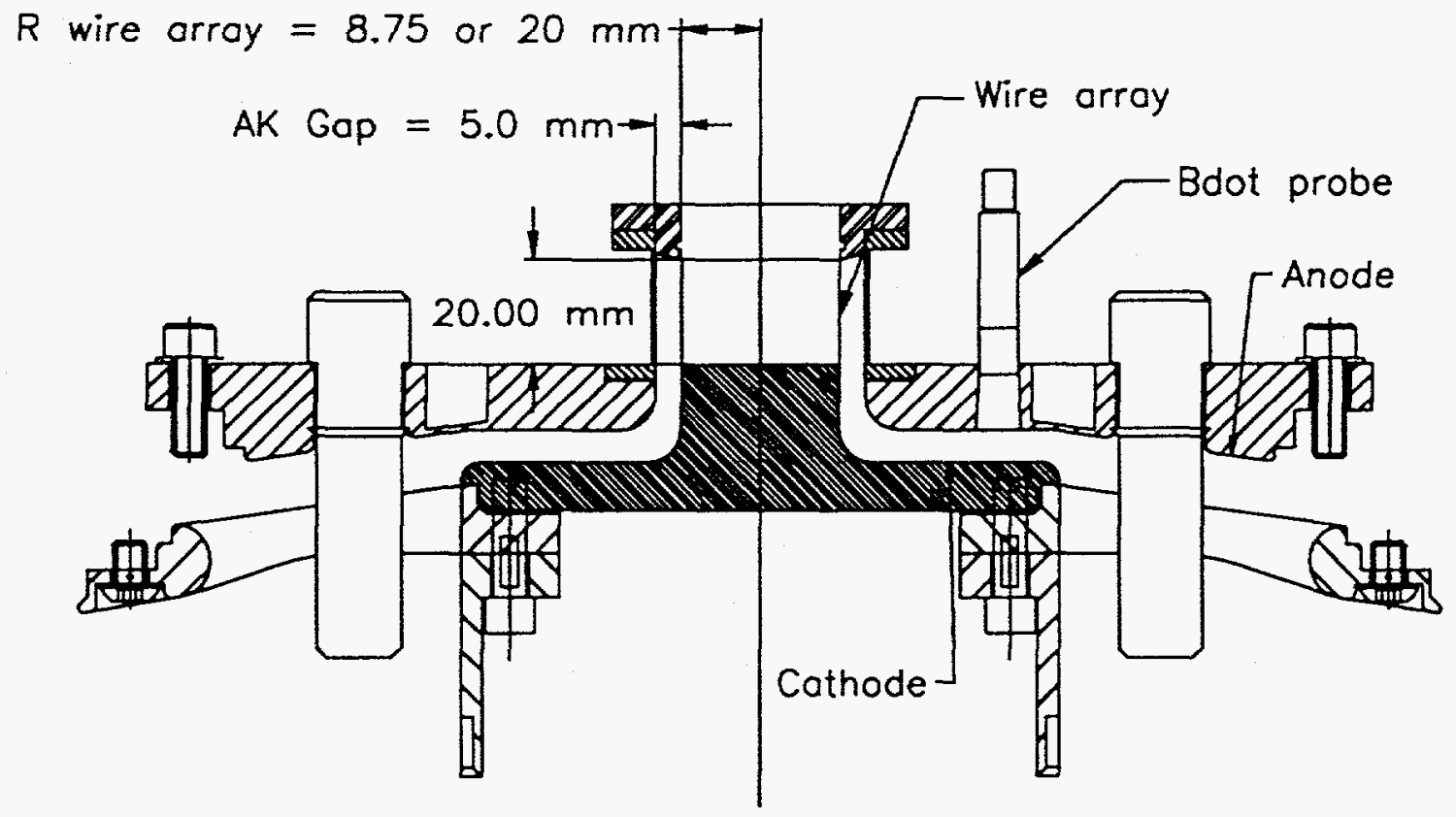

1A. Schematic of wire-array assembly showing the 6-mm radial-feed gap narrowing to the 5-mm anodecathode gap adjacent to the wires and indicating the location of the load-current monitor (B-dot probe).

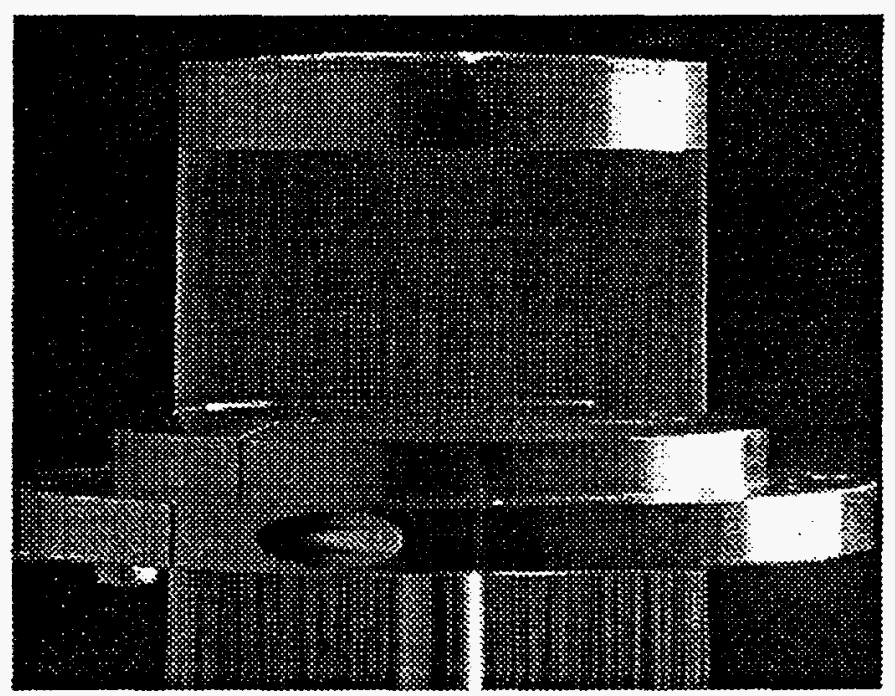

1B. Photograph of one of the $20-\mathrm{mm}$ radius arrays with 240 wires. 

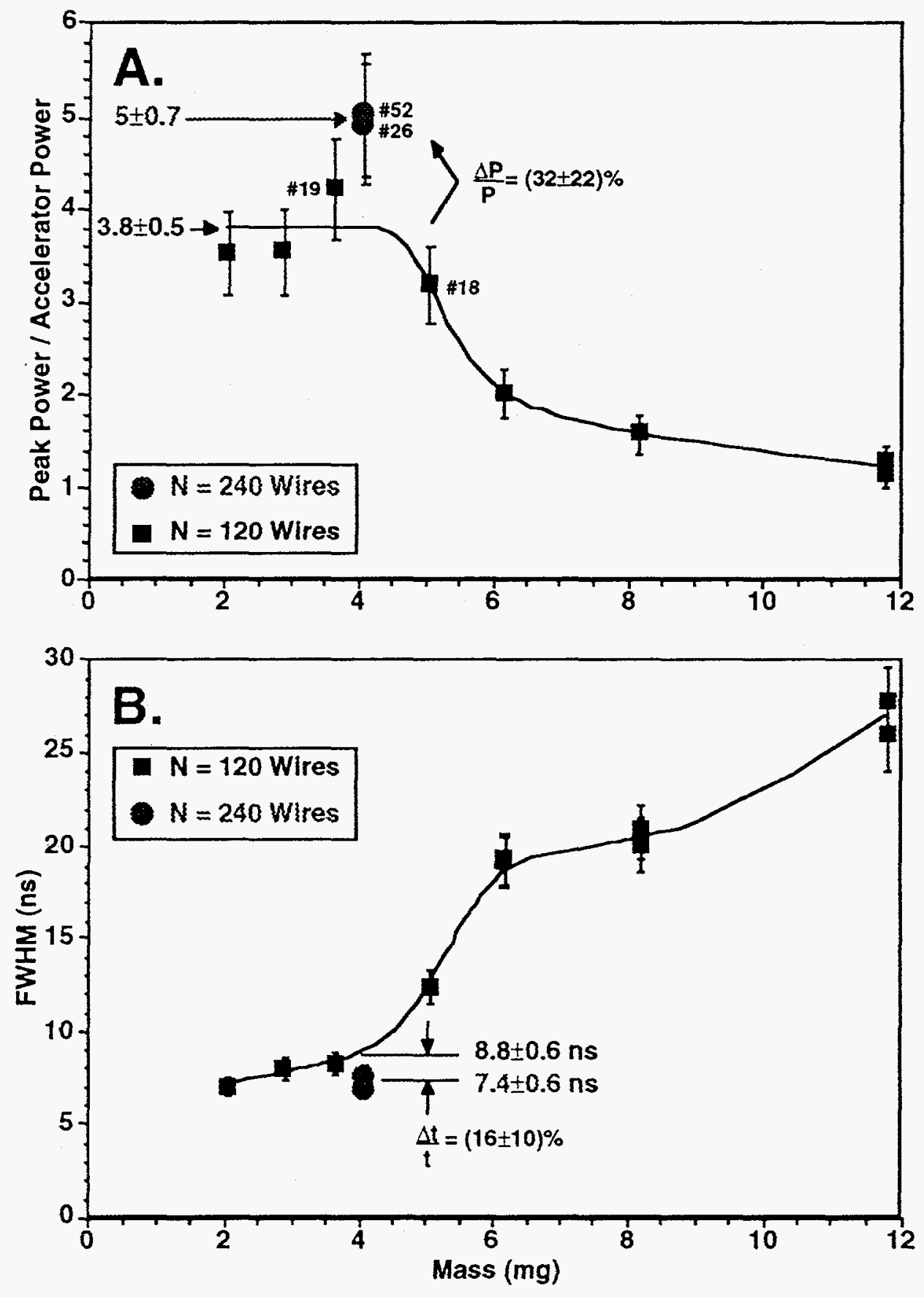

2(A) Ratio of peak total radiated power relative to peak electrical power measured at the insulator stack as a function of array mass, showing increase in radiated power near $4 \mathrm{mg}$ when the number of wires is doubled, for the 20-mm radius array. Shot numbers are shown adjacent the shots near array masses of $4 \mathrm{mg}$. (B) Radiation pulse width as a function of array mass, showing decrease in pulse width (FWHM) near $4 \mathrm{mg}$ when the number of wires is doubled, for the 20-mm radius array. 

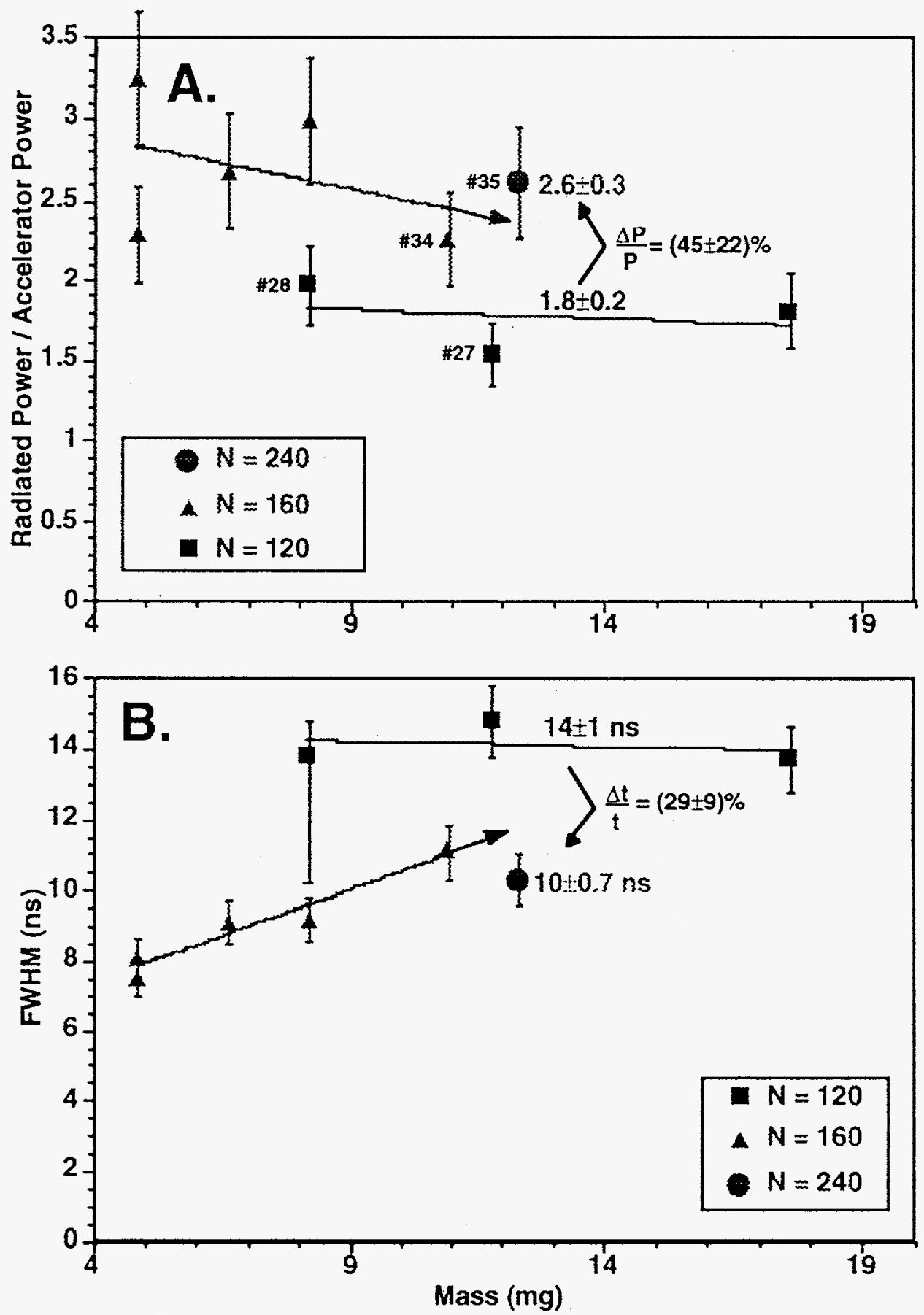

3(A) Ratio of peak total radiated power to peak electrical power measured at the insulator stack as a function of array mass, showing increase in radiated power when the number of wires is increased, for the $8.75 \cdot \mathrm{mm}$ radius array. Shot numbers are shown adjacent selected shots. (B) Radiation pulse width (FWHM) as a function of aray mass, showing decrease in pulse width when the number of wires is increased, for the $8.75-\mathrm{mm}$ radius array. 

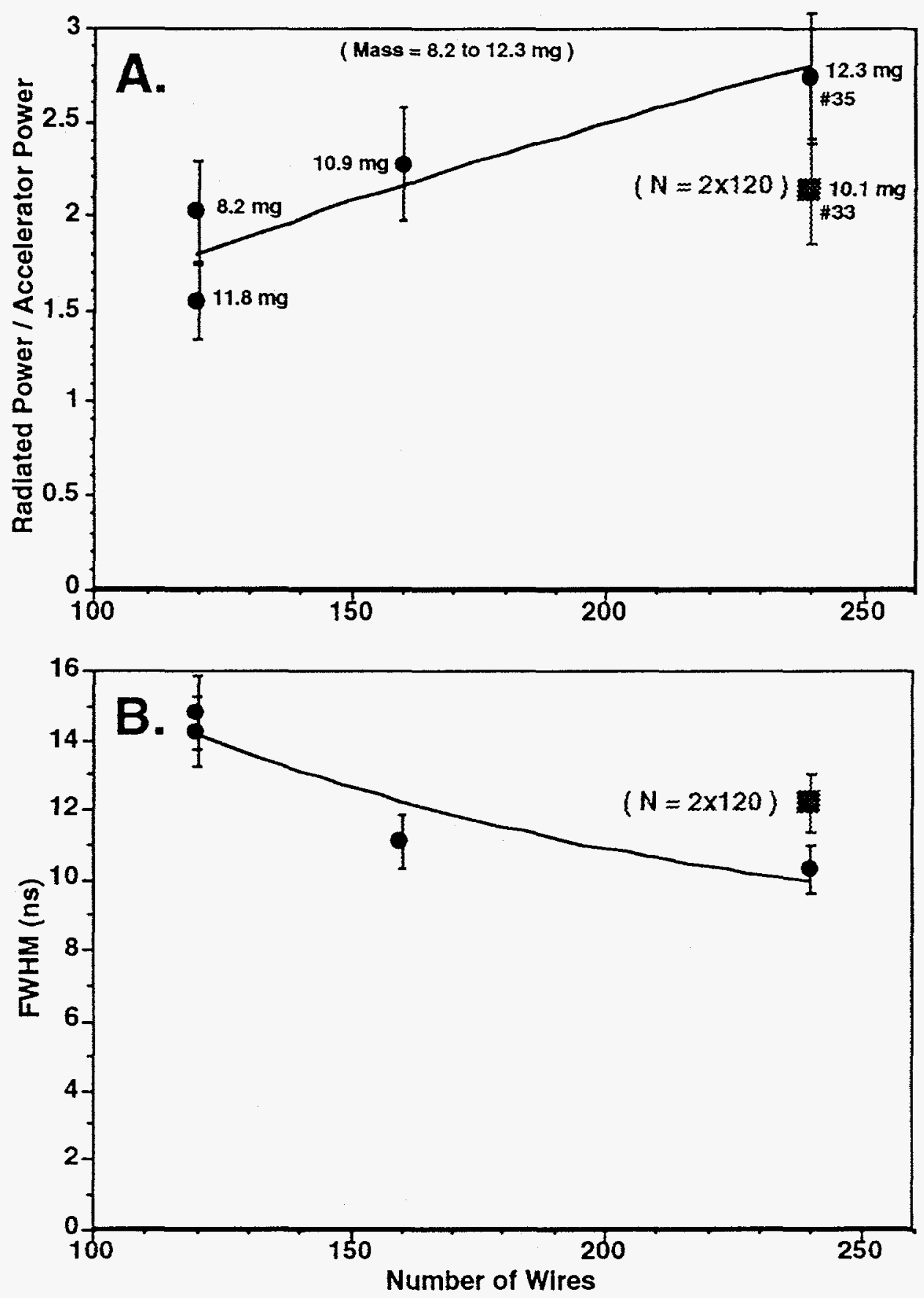

4(A) Ratio of peak total radiated power to peak electrical power measured at the insulator stack as a function of wire number for the $8.75-\mathrm{mm}$ radius array, showing increase in radiated power with wirenumber. Shot numbers are shown adjacent selected shots. (B) Radiation pulse width (FWHM) as a function of wire number for the $8.75-\mathrm{mm}$ radius array, showing decrease in width with wire-number. 

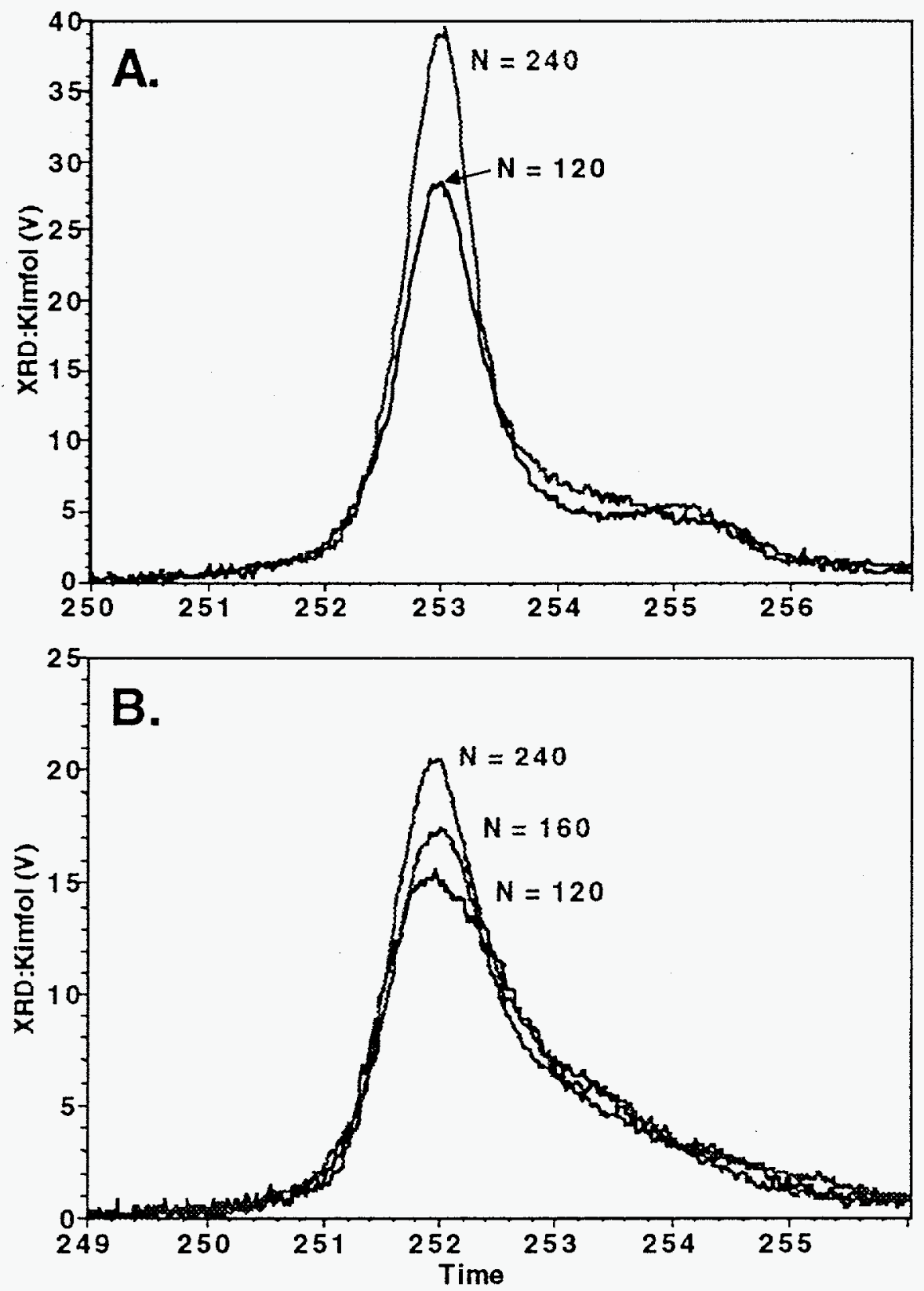

5(A) Comparison of the signal from the XRD filtered by $5 \mu \mathrm{m}$ of Kimfol, for the 120-wire Shot \#19, with that measured for the 240-wire Shot \#26, for an amay mass of about $4 \mathrm{mg}$, showing the increase in peak power and decreasing pulse width with wire number doubling. (B) Comparison of the signal from the XRD filtered by $5 \mu \mathrm{m}$ of Kimfol for the 120-wire Shot \#27, with that measured for the 160wire Shot \#34, with that measured for the 240-wire Shot \#35, at nearty identical array masses of 12 $\mathrm{mg}$, showing the increase in peak power and decreasing pulse width with wire number increase. 

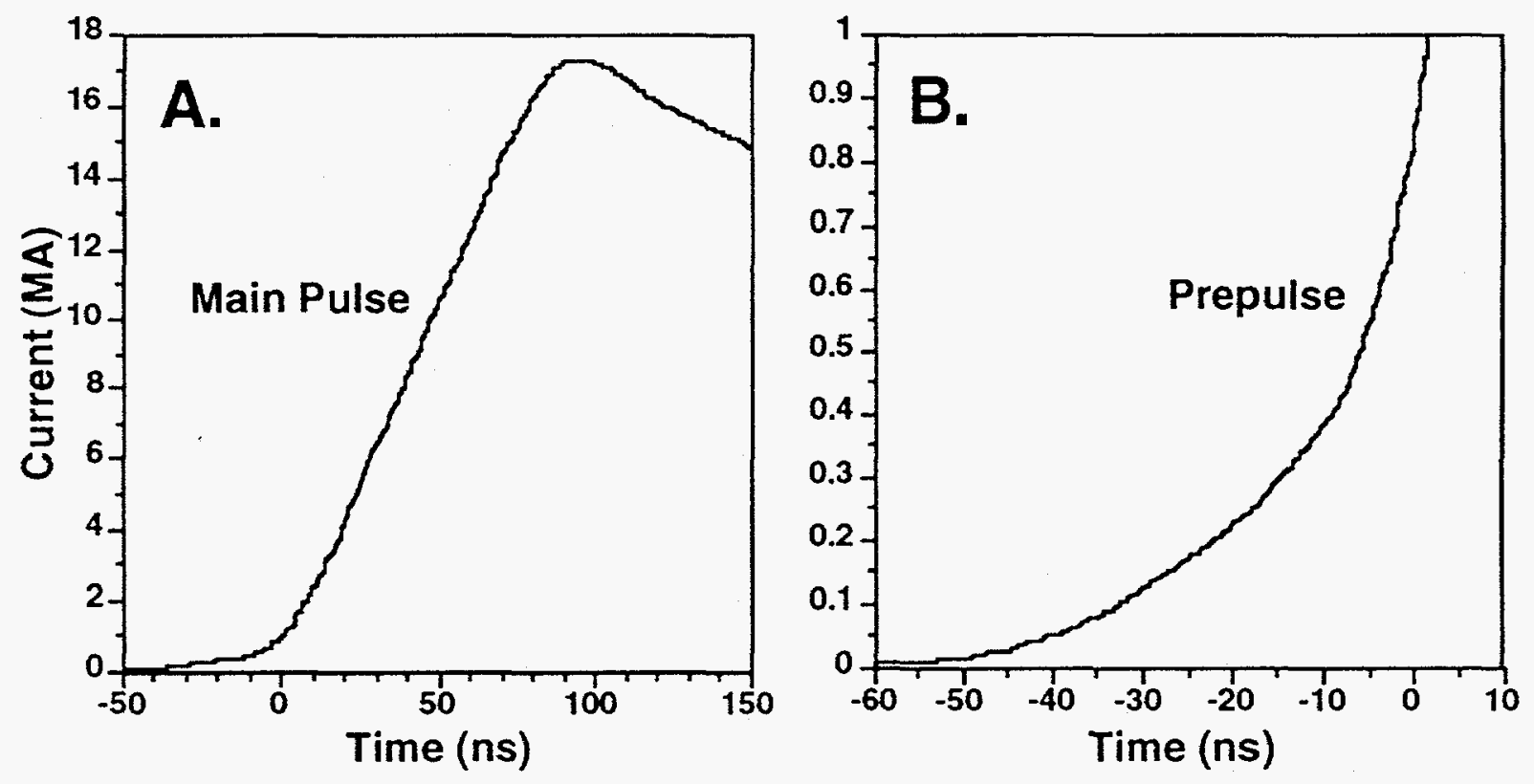

6(A) Main load-current pulse measured for Shot \#18, using B-dot probe shown in Fig. 1A. (B) Loadcurrent prepulse measured for Shot \#18, using B-dot probe shown in Fig. 1A. 


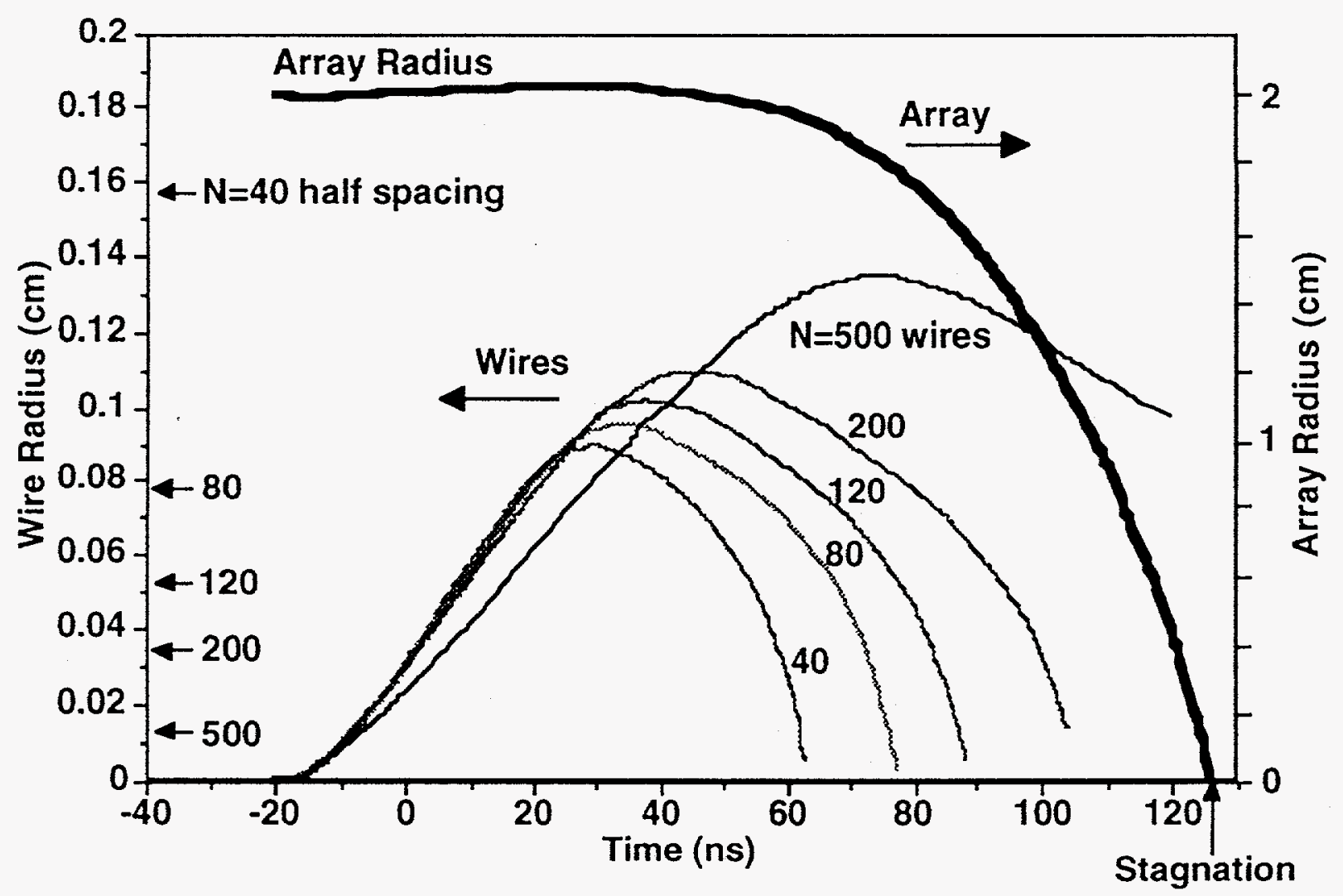

7. Calculated outside radius of the array as a function of time, for Shot \#18 (for array mass of $5.1 \mathrm{mg}$ and initial array radius of $20 \mathrm{~mm}$ ), showing that the implosion of the array starts at about $50 \mathrm{~ns}$. Shown also are individual wires radii calculated as a function of time that correspond to arrays with varying numbers of wire, assuming that the total mass of the array and the initial array radius remains fixed at $5.1 \mathrm{mg}$ and $20 \mathrm{~mm}$. The marks on the left side represent the half spacing for the respective wire numbers. 


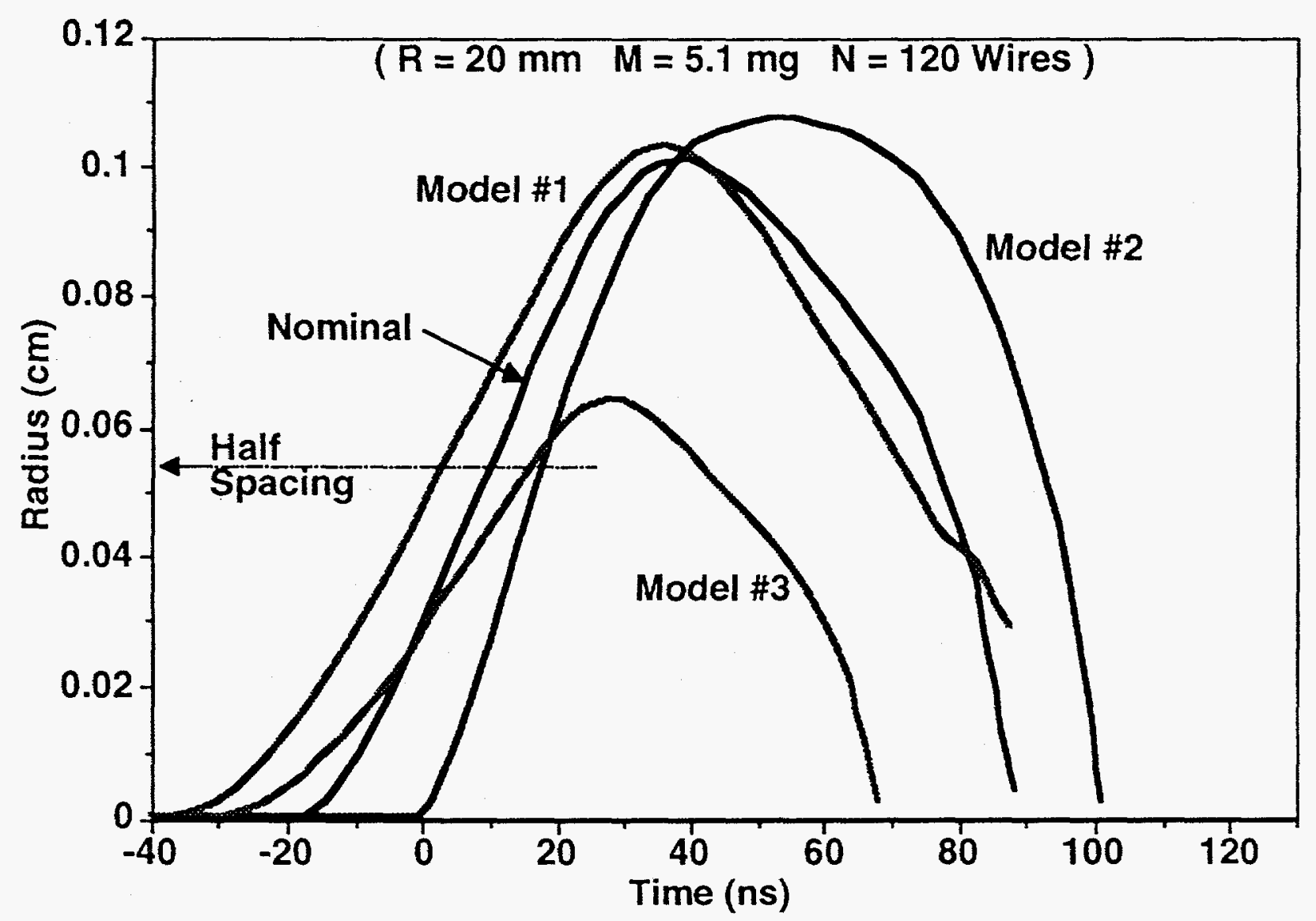

8. Calculated individual wire radii as a function of time and resistivity model (see text) for a 120 wirenumber, $5.1-\mathrm{mg}$-mass, and $20-\mathrm{mm}$-radius array, showing that the results are insensitive to resistivity model except for Model \#3. Half spacing between wires corresponds to $0.52 \mathrm{~mm}$. 